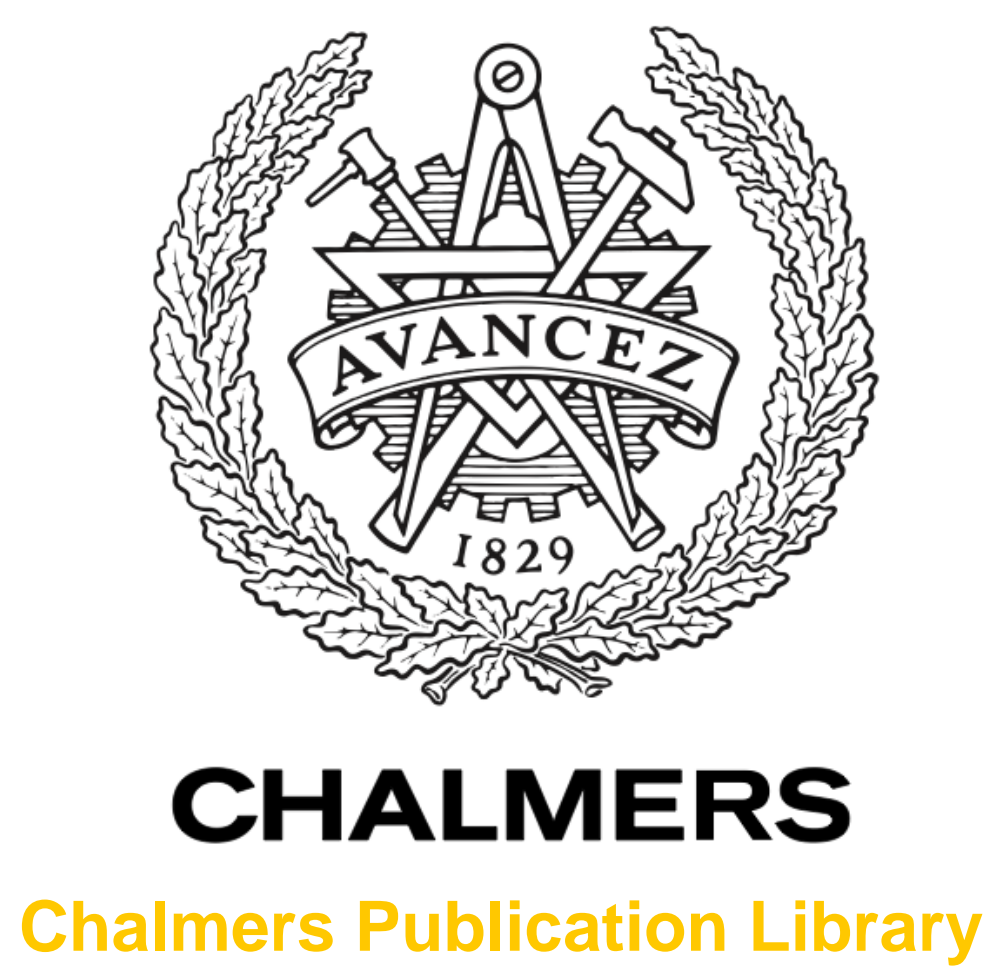

\title{
Self-demodulation effect on subharmonic response of ultrasound contrast agent
}

This document has been downloaded from Chalmers Publication Library (CPL). It is the author's version of a work that was accepted for publication in:

Progress in Biomedical Optics and Imaging - Proceedings of SPIE. Medical Imaging 2012: Ultrasonic Imaging, Tomography, and Therapy, San Diego, CA, 5 - 6 February 2012 (ISSN: 1605-7422)

Citation for the published paper:

Daeichin, V. ; Faez, T. ; Needles, A. (2012) "Self-demodulation effect on subharmonic response of ultrasound contrast agent". Progress in Biomedical Optics and Imaging Proceedings of SPIE. Medical Imaging 2012: Ultrasonic Imaging, Tomography, and Therapy, San Diego, CA, 5 - 6 February 2012, vol. 8320

http://dx.doi.org/10.1117/12.912461

Downloaded from: http://publications.lib.chalmers.se/publication/158198

Notice: Changes introduced as a result of publishing processes such as copy-editing and formatting may not be reflected in this document. For a definitive version of this work, please refer to the published source. Please note that access to the published version might require a subscription. 


\title{
Self-demodulation effect on subharmonic response of ultrasound contrast agent
}

\author{
V. Daeichin ${ }^{1,2}$, T. Faez ${ }^{1}$, A. Needles ${ }^{3}$, G. Renaud ${ }^{1}$, J.G. Bosch ${ }^{1}$, A.F.W. van der Steen ${ }^{1,4}$ and N. de \\ Jong ${ }^{1,4}$ \\ ${ }^{1}$ Biomedical Engineering, Thorax Center, Erasmus MC, Rotterdam, The Netherlands \\ ${ }^{2}$ Department of Signal \& Systems, Chalmers University of Technology, Gothenburg, Sweden \\ ${ }^{3}$ VisualSonics Inc, Toronto, Canada \\ ${ }^{4}$ Interuniversity Cardiology Institute of the Netherlands, Utrecht, The Netherlands
}

\begin{abstract}
In this work the use of the self-demodulation (S-D) signal as a mean of microbubble excitation at the subharmonic (SH) frequency to enhance the SH emission of ultrasound contrast agent (UCA) is studied. SH emission from the UCA is of interest since it is produced only by the UCA and is free of the artifacts produced in harmonic imaging modes. The S-D wave is a low-frequency signal produced by nonlinear propagation of an ultrasound wave in the medium. Single element transducer experiments and numerical simulations were conducted at $10 \mathrm{MHz}$ to study the effect of the S-D signal on the SH response of the UCA by modifying the envelope of the excitation bursts. For 6 and 20 transmitted cycles, the SH response is increased up to $25 \mathrm{~dB}$ and $22 \mathrm{~dB}$ because of the S-D stimulation for a burst with a rectangular envelope compared with a Gaussian envelope burst. Such optimized excitations were used in an array-based micro-ultrasound system (Vevo 2100, VisualSonics Inc., Toronto, ON, Canada) at $18 \mathrm{MHz}$ for in vitro validation of SH imaging. This study suggests that a suitable design of the envelope of the transmit excitation to generate a S-D signal at the SH frequency can enhance the SH emission of UCA and real-time SH imaging is feasible with shorter transmit burst (6cycle) and low acoustic pressure $(\sim 150 \mathrm{KPa})$ at high frequencies $(>15 \mathrm{MHz})$.
\end{abstract}

Keywords: Subharmonic imaging, self-demodulation, ultrasound contrast agent, contrast imaging, high frequency ultrasound.

\section{INTRODUCTION}

The ability to exclusively image blood vessels and blood flow is clinically advantageous for a variety of diagnostic applications. For example, neovasculature within plaques in atherosclerosis has been shown to be a marker of plaque vulnerability [1]. Moreover, using contrast-enhanced ultrasound imaging for real-time visualization of tissue perfusion is another important indicator of malignancy or physiological failures [2]. Besides the safe, inexpensive, and portable nature of ultrasound imaging, its ability for real-time imaging is perhaps one of the biggest advantages of this modality for preclinical and clinical applications [2].

Ultrasound contrast agents (UCA) are micron size gas bubbles encapsulated by lipid, protein, albumin or polymer shell. They enhance the contrast of blood pool for ultrasound imaging. When exposed to ultrasound, the microbubbles behave as a nonlinear mechanical resonator whereas tissue scattering is linear. The result of such a nonlinear behavior is the transfer of energy to harmonic multiples of the transmit frequency $\left(\mathrm{f}_{0}\right)$. Applying linear contrast detection techniques such as post-processing subtraction algorithms, echoes from UCA could, in certain conditions, be visualized against background tissue echoes. Despite of not being real-time, subtraction techniques are not successful in UCA detection under many imaging conditions such as for tissue perfusion in small capillaries or in the presence of breathing and cardiac-induced motions [3]. The nonlinear signals of UCA have been used in different techniques such as harmonic imaging [4] and pulse inversion (PI) [5] to achieve more sensitive contrast detection techniques while suppressing the tissue backscatter echoes. In addition, using such methods provides real-time imaging of the UCA.

Medical Imaging 2012: Ultrasonic Imaging, Tomography, and Therapy, edited by Johan G. Bosch,

Marvin M. Doyley, Proc. of SPIE Vol. 8320, 83200W · (c) 2012 SPIE

CCC code: $1605-7422 / 12 / \$ 18 \cdot$ doi: $10.1117 / 12.912461$

Proc. of SPIE Vol. $832083200 \mathrm{~W}-1$ 
In general, nonlinear imaging approaches are thought to perform best when the bubbles are insonified at or near their resonance frequency [6]. The resonance frequency of conventional UCA bubbles ranges generally between $1 \mathrm{MHz}$ and 8 $\mathrm{MHz}$ [7], which is therefore well-suited for the majority of diagnostic ultrasound applications. There is, however, increasing interest in employing higher transmit frequencies (15 to $50 \mathrm{MHz}$ ), for applications requiring a higher spatial resolution such as small animal imaging [3], carotid and intravascular coronary vasa vasorum imaging [8]. Under these circumstances the performance of nonlinear imaging techniques is degraded because the driving frequency is higher than the resonance frequency. Moreover second harmonic imaging techniques are hampered because of the increase in the amplitude of harmonics produced by tissue as a result of nonlinear propagation of ultrasound.

The emission of energy at subharmonic $(\mathrm{SH})\left(0.5 \mathrm{f}_{0}\right)$ and ultraharmonic $\left(1.5 \mathrm{f}_{0}, 2.5 \mathrm{f}_{0}\right.$, etc) frequencies by UCA was proposed to be exploited as a new imaging modality [9]. No SH signal is generated during propagation in tissue and no SH scattering is produced by tissue. The SH signal has a greater penetration distance since its attenuation is lower than that at the fundamental frequency, offering a potentially powerful diagnostic tool for clinical examinations [2]. Unlike the second and higher harmonics amplitudes that increase monotonically (for moderate acoustic pressures) with the amplitude of the incident wave [10], the SH response has a threshold behavior [11]. This threshold exhibits two minima: when the frequency of the excitation signal is close to the resonance frequency of the microbubble and close to twice the resonance frequency which is usually a lower minimum than the first one [12]. The SH signal is strongly dependent on the applied acoustic pressure, the hydrostatic pressure variations [13] and the envelope of the excitation signal [14].

A good contrast imaging technique such as SH imaging keeps the nonlinear signal of the UCA while completely suppresses the tissue backscatter signal. On the other hand, the fundamental imaging provides anatomical landmarks for sonographic navigation and anatomy related to the perfusion. Thus, having a side by side real-time fundamental and $\mathrm{SH}$ images would allow identification of anatomical structures, while also simultaneously providing the SH information on vascular structures and positions of UCAs. Eisenbrey et al. demonstrated the possibility of such simultaneous fundamental and SH imaging on a modified commercial scanner at low frequencies (2-4 MHz) [2].

Initial attempts to detect UCA at high frequencies used nonlinear harmonic imaging in single element, mechanically scanned transducers systems [15]. The fixed focus of transducers, low maximum penetration depths and mechanical motion of the transducers were some of the limitations of such systems for detecting UCA at high frequencies [3]. Later on an integrated array-based micro-ultrasound imaging system was developed by Foster et al. [16]. It has a 64-channel, high-frequency beamformer, capable of driving linear arrays in the 15-50 MHz range. Compared with single-element transducer systems, such a linear array has higher depth-of-field, allowing more flexibility in pulsing schemes, better UCA detection over larger depth ranges and improved overall image quality [3]. Using such array based high frequency system Needles et al tried to determine the optimal imaging strategy for a real-time nonlinear contrast mode and validate the optimal strategy in vivo by generating parametric images based on nonlinear contrast detection [3]. They studied different conventional nonlinear contrast imaging methods such as PI, amplitude modulation (AM) and combination of $\mathrm{AM}$ and PI (AMPI) at both fundamental and SH frequencies. Transmitting waves with rectangular envelopes they found $15 \mathrm{~dB}$ enhancement in contrast to tissue ratio (CTR) for pulse inversion (PI) with $\mathrm{SH}$ imaging compared with the fundamental imaging. However no explanation for such enhancement in the CTR was provided in that study.

In this study, we investigate the effect of the self-demodulation (S-D) signal on the SH response of phospholipid-coated microbubbles excited at $10 \mathrm{MHz}$ using both single element transducer experiments and numerical simulations. Optimized excitation bursts were then used together with conventional nonlinear contrast detection methods such as PI and AM in a commercial high frequency array based ultrasound system for in vitro validation. Our hypothesis is that the enhancement of the SH signal using an excitation wave with a rectangular envelope can be explained by the S-D stimulation effect on the SH response of the UCA. We show that understanding the effect of the S-D signal on the UCA behavior can help to optimize the envelope of the excitation signal for SH imaging especially at high frequencies. In vivo, a chicken embryo model was used for real time side by side fundamental and SH imaging at $18 \mathrm{MHz}$.

\section{NUMERICAL SIMULATIONS}

\subsection{Self-demodulation signal}


In a weakly nonlinear regime the propagation of an ultrasound pulse gives rise to a low-frequency signal due to the S-D phenomenon. The S-D pressure wave, $\mathrm{P}_{\mathrm{sd}}(\mathrm{t})$, is related to the envelope of the transmitted signal, $\mathrm{E}(\mathrm{t})$, as follows [17 and 18]:

$$
p_{s d}(t) \propto \frac{\partial^{2} E^{2}(t)}{\partial t^{2}}
$$

The envelope function of the transmit burst used in this study is:

$$
E(t)=e^{-\left(\frac{2 t}{T}\right)^{2 M}}
$$

where $\mathrm{T}$ is the duration of the signal and the integer $\mathrm{M}$ determines the rise and decay time of the envelope. For $\mathrm{M}$ equal to 1 this function represents a Gaussian envelope and increasing the value of $\mathrm{M}$ results in a more rectangular shape envelope. Calculating the nonlinear propagation of a collimated ultrasound beam, we used the equation derived by Khokhlov, Zabolotskaya, and Kuznetsov (KZK) [19]. It has been shown that such a model can accurately simulate the S$\mathrm{D}$ of a sine burst [17 and 18]. A transducer focused at $76 \mathrm{~mm}$ with a diameter of $10 \mathrm{~mm}$ was used as an excitation source. The KZK model was adopted for our simulation as implemented by Vos et al [18]. The results of the KZK simulations to estimate the nonlinear propagation of a $10 \mathrm{MHz}$ ultrasound pulse, for three different envelopes $(\mathrm{M}=1 ; 3 ; 15$ and $\mathrm{T}=0.6$ $\mu \mathrm{s})$ are shown in figure 1. The transmitted acoustic peak pressure at focus is $50 \mathrm{kPa}$. For $\mathrm{M}=1$ (Gaussian envelope), the $\mathrm{S}-\mathrm{D}$ signal appears as a peak around $1 \mathrm{MHz}$ in the frequency domain (figure $1 \mathrm{c}, \mathrm{M}=1$ ). For $\mathrm{M}=3$ (figure $2 \mathrm{c}, \mathrm{M}=3$ ) a more distributed S-D signal with center frequency around $2 \mathrm{MHz}$ is obtained. Increasing $\mathrm{M}$ to higher values (e.g. $\mathrm{M}=15$ ) results in the transfer of energy to a broader range of low frequencies until no distinct peak can be seen for the S-D signal in the frequency domain (figure $2 c, M=15$ ). For both $M=3$ and $M=15$ the $S-D$ signals are broad-band low frequency signals which overlap with the side lobes of the fundamental frequency peaks $(10 \mathrm{MHz})$. Although increasing the value of $\mathrm{M}$ to 15 in the envelope function results in a more broad-band low frequency S-D signal, comparing the frequency content of the propagated waves (figure 1c) indicates that the transmitted pulse with a rectangular envelope $(\mathrm{M}=15)$ has the highest S-D energy around the SH frequency $(5 \mathrm{MHz})$.
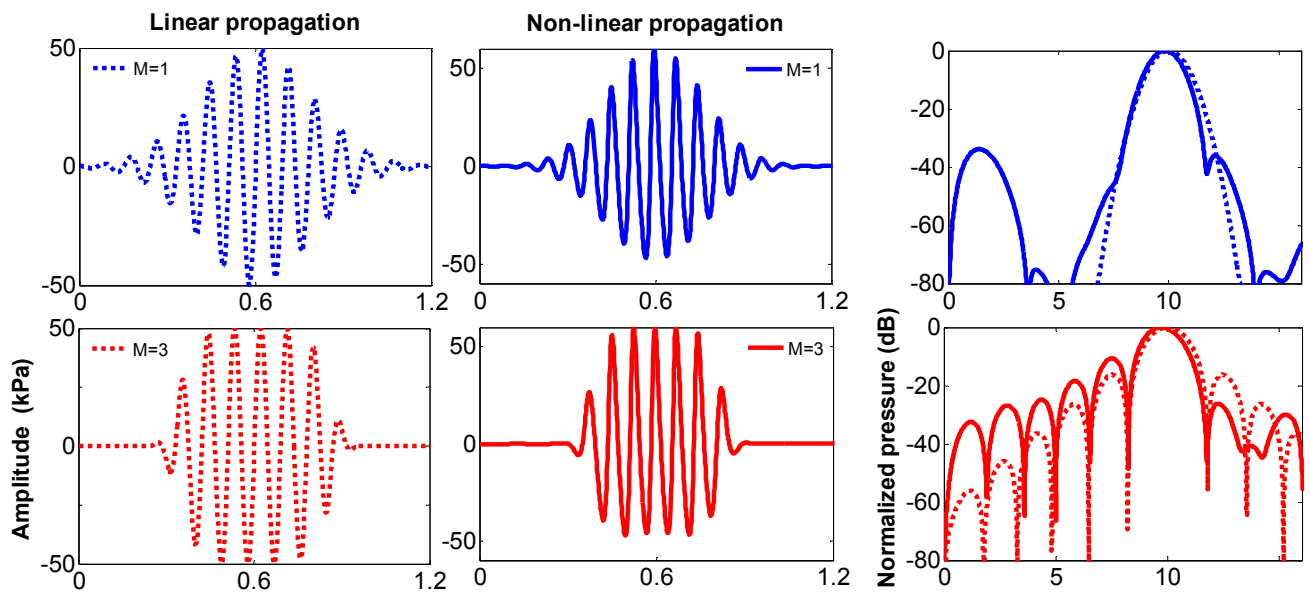

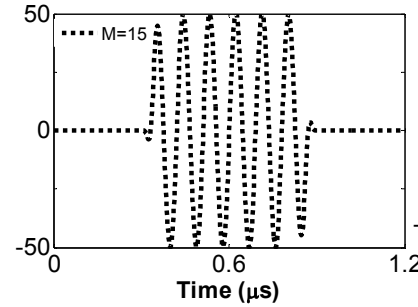

a

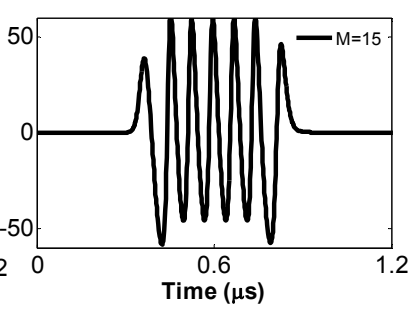

b

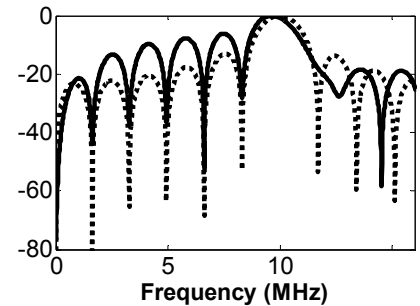

C

Figure 1. Propagation of a $10 \mathrm{MHz}$ burst with three different envelopes ( $\mathrm{M}=1,3$ and 15; $\mathrm{T}=0.6 \mu \mathrm{s})$ using the KZK model [6] showing the waves a) after linear propagation, b) after non-linear propagation, and c) their corresponding frequency spectra. 


\subsection{Effect of the self-demodulation signal on the subharmonic emission of a microbubble}

We linked the KZK model [6] to the Marmottant model [20], which describes the dynamics of lipid-coated microbubbles, in order to investigate the influence of the S-D signal on the SH response of the UCA. The KZK model calculates the pressure wave after nonlinear propagation. Such calculated pressure wave is then used as an excitation wave in the Marmottant model in order to compute the radial response of a microbubble.

To see the effect of the S-D signal on the SH enhancement we investigated the amplitude of the SH oscillation of a single microbubble with an initial surface tension $\left(\sigma\left(R_{0}\right)\right)$ of $0.01 \mathrm{~N} / \mathrm{m}$ in response to three different excitation bursts (figure 2). The shell elasticity $(\chi)$ and the shell viscosity $\left(\kappa_{s}\right)$ were set to $2.5 \mathrm{~N} / \mathrm{m}$ and $10^{-9} \mathrm{~kg} / \mathrm{s}$, values for BR14 UCA (Bracco Research SA, Geneva, Switzerland) used for in vitro experiments [21]. The diameter of the microbubble was set to $2 \mu \mathrm{m}$ which is also the mean diameter of BR14 UCA [22]. It can be seen from figure 2 that the SH response of such a bubble is enhanced up to $23 \mathrm{~dB}$ by increasing the $\mathrm{M}$ in the envelope function of the excitation burst from 1 to 15 .
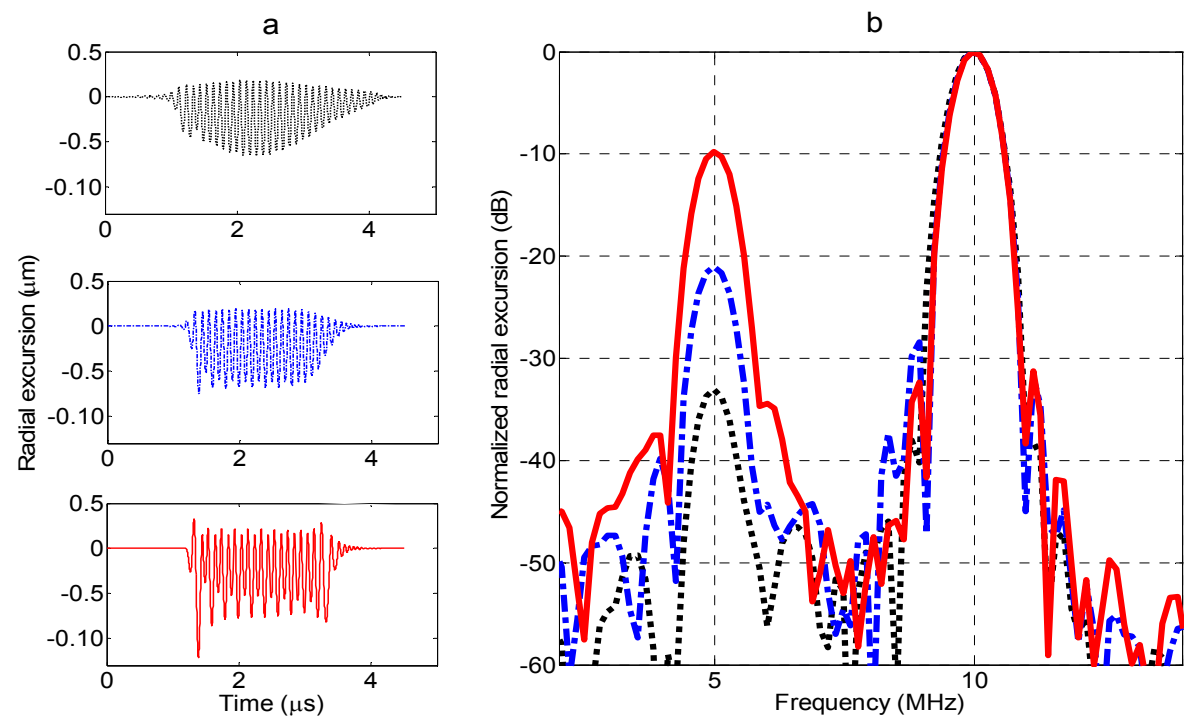

Figure 2. Simulated radial excursion of a $2 \mu \mathrm{m}$ microbubble excited by a 20-cycle burst with three different envelopes after nonlinear propagation (at the focus): $\mathrm{M}=1$ (dashed-dotted curve), $\mathrm{M}=3$ (dashed curve) and $\mathrm{M}=15$ (solid curve) and their corresponding amplitude spectra for an initial surface tension of $0.01 \mathrm{~N} / \mathrm{m}$.

\section{SINGLE ELEMENT TRANSDUCER EXPERIMENTS}

\subsection{Materials and methods}

A $10 \mathrm{~mm}$ diameter thin shell cylindrical tube having a total thickness of $52 \mu \mathrm{m}$ was mounted in the center of a triangular water tank at the focus of two transducers used for transmitting and receiving the ultrasound signals. The tube was filled with distilled, saturated water. BR14 microbubbles were reconstituted to manufacturer's specifications and diluted 10,000 times then poured in the tube and mixed gently using a magnetic stirrer in order to have a homogeneous suspension. The microbubbles consist of a phospholipid monolayer shell with a perfluoropropane gas core. A broad-band transducer with centre frequency of $10 \mathrm{MHz}$ and $76 \mathrm{~mm}$ focal length, (Panametrics V311; 3-13 MHz) was used for transmit. The transducers were calibrated using a $0.2 \mathrm{~mm}$ PVDF probe hydrophone in a separate experiment (Precision Acoustics Ltd., Dorchester, UK). The echoes from the microbubbles were received at half the transmitting frequency (5 $\mathrm{MHz}$ ) by another focused transducer with the same focal length (Precision Acoustics PA076; 1-9 MHz). Acoustic pressure amplitudes of $50 \mathrm{kPa}$ were applied. The transmitted signals were generated by an arbitrary wave generator (8026, Tabor Electronics Ltd., Tel Hanan, Israel) and amplified with a $60 \mathrm{~dB}$ pulse amplifier (150A100B; Amplifier Research, Limerick, Ireland). The scattered pressure waves were received, amplified by $60 \mathrm{~dB}$ and digitized by a 12-bit digitizer with a sampling frequency of $500 \mathrm{MHz} .10 \mathrm{MHz}$ bursts of 6 and 20 cycles were transmitted. The envelope of the transmit burst was varied from the Gaussian to the rectangular shape by changing the value of M to 1, 3 and 15 (Eqn. 
2). Averaging over 50 pulses in the frequency domain with a pulse repetition frequency of $1 \mathrm{kHz}$ was applied, and this sequence was repeated for each of the transmitted signals with different envelopes.

\subsection{Single element transducer experimental results}

Figure 3 shows the signals scattered by the microbubbles in response to a 6-cycle and a 20-cycle $10 \mathrm{MHz}$ bursts with three envelopes changing from a Gaussian shape $(M=1)$ to a more rectangular shape $(M=15)$. For the 20-cycle excitation the energy of the $\mathrm{SH}$ response produced by the UCA increases from $-55 \mathrm{~dB}$ to $-33 \mathrm{~dB}$ for $\mathrm{M}=1$ to $\mathrm{M}=15$, thus a $22 \mathrm{~dB}$ enhancement is observed (figure $3 \mathrm{~b}$ ). Almost the same enhancement ( $25 \mathrm{~dB})$ is observed For the excitation bursts with 6cycle for $\mathrm{M}=15$ compared with $\mathrm{M}=1$ (figure $3 \mathrm{a}$ ).
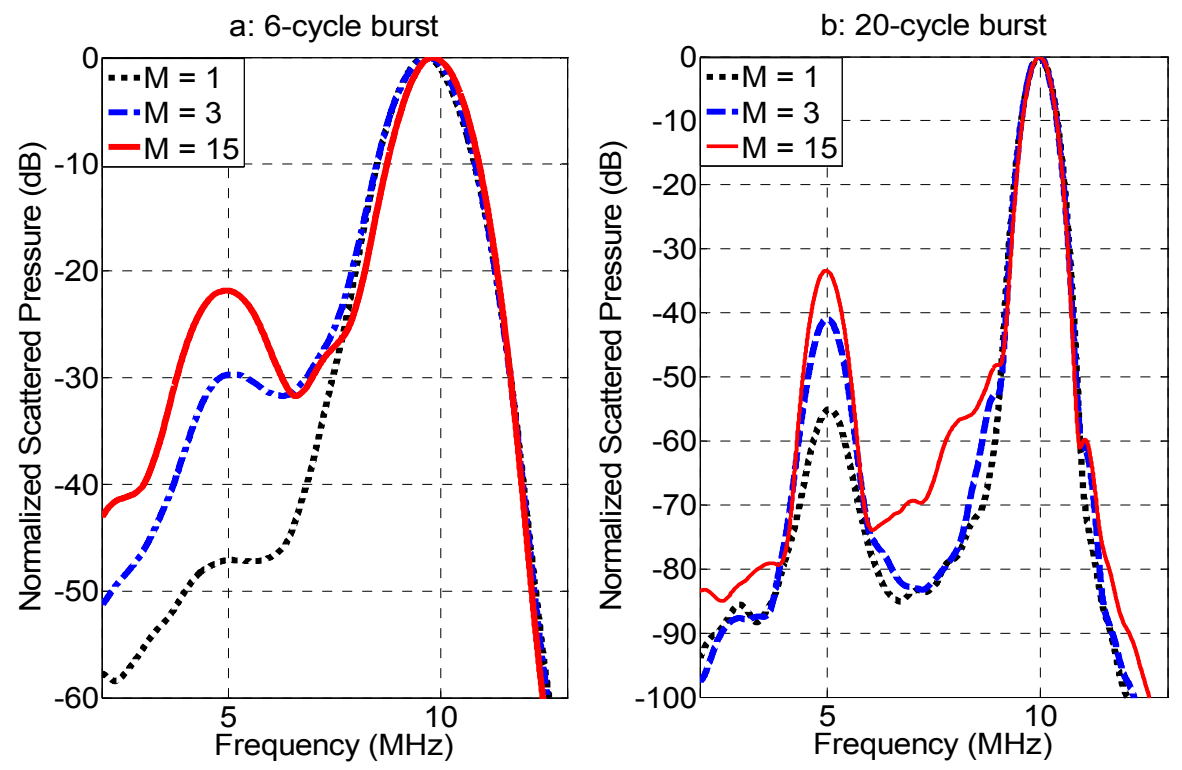

Figure 3. The amplitude spectrum of the scattered signal from the microbubbles (average of 50 acquisitions in the frequency domain) excited by a $10 \mathrm{MHz}, 50 \mathrm{kPa}$ peak pressure burst with three different envelopes a) 6-cycle burst; b) 20-cycle burst.

\section{HIGH FREQUENCY CONTRAST IMAGING}

\subsection{Instrumentation}

A high frequency pre-clinical ultrasound scanner (Vevo 2100, VisualSonics Inc., Toronto, ON, Canada) was operated at $18 \mathrm{MHz}$, with a 256-element linear array transducer (model MS-200). low-amplitude ( 150 kPa) narrowband 20-cycle transmit pulses with rectangular envelope were transmitted with the following pulse sequences: B-mode, PI and AM. The received echoes were analyzed using system temporal filters providing real-time band-pass filtering at the SH and fundamental frequencies.

The MicroMarker (VisualSonics Inc., Toronto, Canada) UCA was used in this study. These microbubbles consist of a phospholipid shell encapsulating a C4F10/N2 gas core. These are obtained after reconstitution of a lyophilisate cake with $1 \mathrm{~mL}$ of $0.9 \%$ saline, resulting in a concentration of approximately $2 \cdot 10^{9}$ bubbles $/ \mathrm{mL}$, with a mean bubble diameter of $1.2 \mu \mathrm{m}[3]$.

For the in vitro experiments a tissue mimicking phantom was prepared according to the recipe of [23]. During the formation of the phantom a steel rod was placed in the phantom to make a cylindrical wall-less hole with a diameter of $10 \mathrm{~mm}$ in the phantom. A suspension of Micro-Marker was prepared as a 1:10000 dilution in saline after agent reconstitution. The microbubble solution was then poured into the wall-less hole inside the phantom. For maintaining a homogeneous distribution of the UCA a magnetic stirrer was used to mix the bubble solution gently. Identical rectangular region of interest in the same depth, one in the tissue and one in the bubbles, were selected for CTR 
calculations (figure 6). Fast Fourier transforms (FFTs) were applied to compare the levels of nonlinearity at the fundamental and the SH frequencies as well as residual tissue signals (figure 5). Since a rectangular envelope was used for transmit pulse the S-D signal is supposed to be present in all the three different modes. The brightness in the gray scale images were normalized to one maximum value and the dynamic range of 30 was used for displaying the images.

\subsection{In vitro results}

Figure 5 shows the frequency spectra of UCA and tissue-mimicking material from the region of interest for three different pulse sequences. The SH peaks (at $9 \mathrm{MHz}$ ) in the spectra of the UCA backscattered signals are present for all the pulse sequences, however no SH is observed in the backscatter signals of the tissue-mimicking material for any of them. At the fundamental frequency $(18 \mathrm{MHz})$ the maximum amplitude belongs to the signals of both UCA and tissue mimicking phantom in the B-mode. Using the AM, the fundamental signals of UCA and tissue-mimicking material are decreased $5 \mathrm{~dB}$ and $10 \mathrm{~dB}$ respectively compared with the signals in the B-mode. However the PI method reduces the signals at the fundamental frequency to the noise level (15 dB below the amplitude of the signals in the B-mode). The opposite situation accounts for the signal at the SH frequency; PI gives the maximum amplitude whereas AM and Bmode results in $\mathrm{SH}$ peaks with $-3 \mathrm{~dB}$ and $-8 \mathrm{~dB}$ compared to the signal in the PI mode.

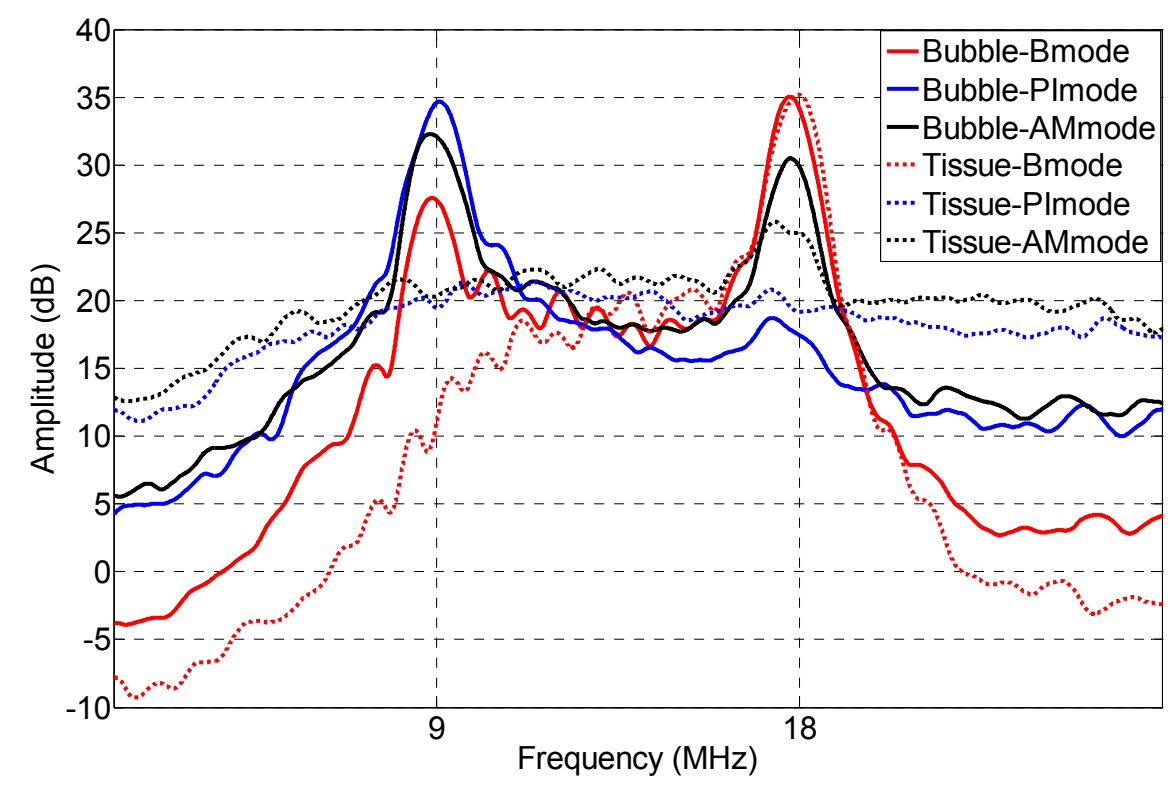

Figure 5. Frequency spectra of UCA (solid) and tissue-mimicking material (dashed) for the different pulse sequences. Bmode (red), PI (blue), AM (black). The transmit frequency was $18 \mathrm{MHz}$.

In vitro comparison between the fundamental B-mode and the SH image with PI is depicted in figure 6 . The calculated CTRs from the regions of interests were $-15 \mathrm{~dB}$ for the b-mode image filtered at fundamental frequency and $13 \mathrm{~dB}$ for the PI image filtered at SH frequency. 

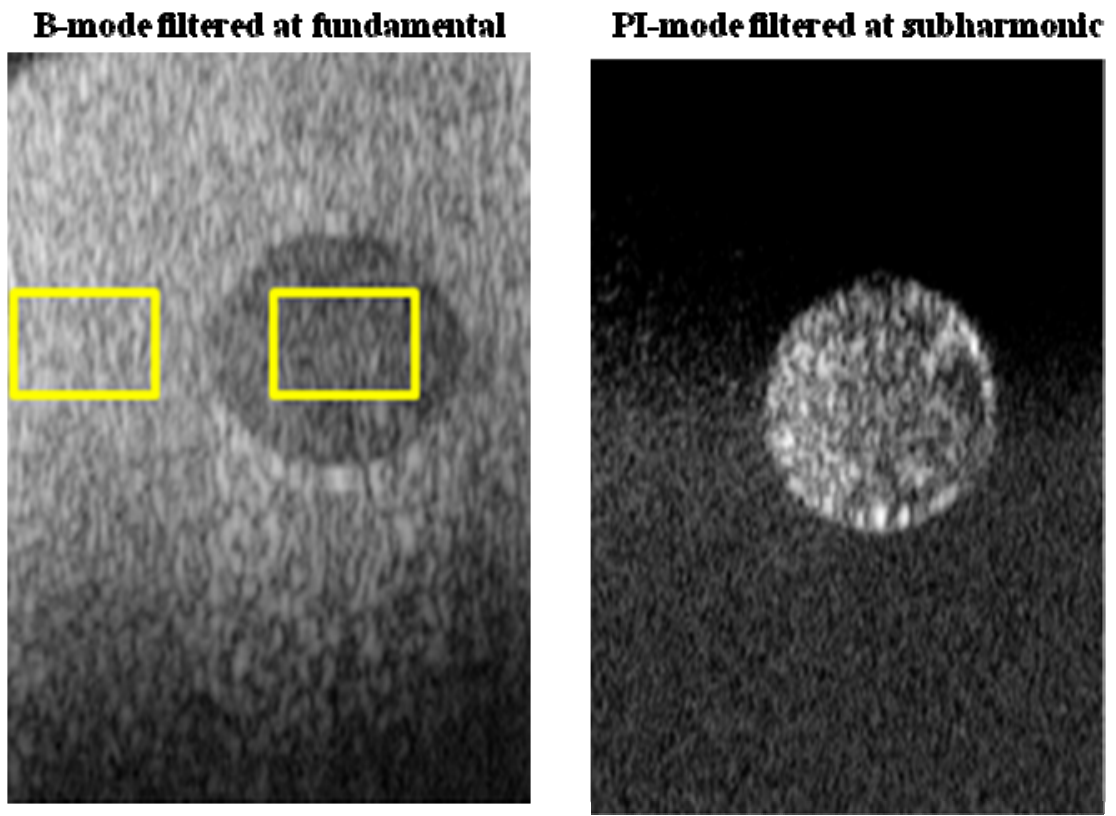

Figure 6. In vitro comparison between the fundamental B-mode and the contrast mode (PI filtered at SH frequency).

\section{DISCUSSION}

\subsection{Effect of the self-demodulation signal on the subharmonic emission of microbubbles}

Our results including numerical simulations and in vitro experiments suggest that an excitation burst with a rectangular envelope contains a broad-band S-D signal which acts as a stimulator and triggers enhanced SH responses of microbubbles. For a $0.6 \mu$ s burst with a Gaussian envelope $(M=1)$, the frequency content of the S-D signal is limited to the very low frequency and it appears as a clear peak around $0.75 \mathrm{MHz}$ (figure $1 \mathrm{c}, \mathrm{M}=1$ ). This peak becomes broader by increasing $\mathrm{M}$ to 3 (figure. 1c). Finally such a clear peak in the frequency domain disappears for an ultrasound burst with a rectangular envelope (figure 1c, M=15). Although the $\mathrm{S}-\mathrm{D}$ signal is not concentrated at a specific low frequency for a burst with a rectangular envelope, it has higher energy at half the transmit frequency than for a Gaussian envelope.

It has been shown before that the initial surface tension is a key parameter in the generation of low amplitude $\mathrm{SH}$ emission by lipid coated microbubbles [21]. According to the model of Marmottant [20], a sharp change in the shell stiffness, occurring when the coating buckles, can enhance the SH response. Our simulation results (data are not presented here) suggest that the stimulation effect of the S-D signal on the SH response of the microbubble is significant when the initial state of the microbubble is close to the buckling regime but not in the buckled state. Far from the buckling regime, the microbubble exhibits no SH oscillation and the S-D signal is not strong enough to "push" the bubble into that regime in the range of acoustic pressure investigated in this study $(50-150 \mathrm{kPa})$. Also, when the bubble is already placed in the buckled state $\left(\sigma\left(\mathrm{R}_{0}\right)=0\right)$ the S-D signal does not enhance the SH emission since it is already in its maximum level. However for intermediate state, the S-D signal in the burst with rectangular envelope has enough energy to "push" the bubble towards the buckling regime and increase the SH response of the microbubble. The enhancement of the SH energy at $\sigma\left(R_{0}\right)=0.01 \mathrm{~N} / \mathrm{m}$ is depicted in figure 2. As can be seen the SH response of a microbubble, thanks to the S-D signal, increases up to $23 \mathrm{~dB}$ by changing the 20 -cycle burst envelope from Gaussian $(\mathrm{M}=1)$ to rectangular $(\mathrm{M}=15)$. A microbubble with a diameter of $2 \mu \mathrm{m}$ was chosen for the numerical simulation since it is reported as the mean diameter of BR14 UCA [22]. The SH resonance frequency of a $2 \mu \mathrm{m}$ BR14 microbubble is around $5 \mathrm{MHz}$ [21] indicating that the excitation frequency $(10 \mathrm{MHz})$ is twice its $\mathrm{SH}$ resonance frequency which is known to give the minimum threshold of SH emission from the UCA [12].

To validate experimentally the stimulating effect of the S-D signal on the SH emission predicted by numerical simulations, scattering measurements were performed on a population of microbubbles. Experimental results (figure. $3 \mathrm{~b}$ ) 
showed that the SH amplitude scattered by the UCA increases monotonically up to $25 \mathrm{~dB}$ and $22 \mathrm{~dB}$ by changing the 6cycle and the 20-cycle burst envelopes from Gaussian $(M=1)$ to rectangular $(M=15)$. The enhancement of the SH signal due to the shape of the excitation bursts in the scattering measurements are in excellent agreement with the simulations (figure. 3). The enhancement of the SH emission because of the envelope of the transmit burst was also reported before [14]. However, a physical explanation for this observation was not provided earlier. We believe that the stimulation effect of a burst having a rectangular envelope is due to its S-D signal as shown in figure 1. The enhancement in the SH response of the UCA caused by the low frequency S-D signal is supported by numerical simulation and experiments performed in this study.

\subsection{Practical implementation for subharmonic imaging}

Short signals are more favored for imaging purposes since they provide a higher spatial resolution. However, with such signals, it is difficult to separate the SH component from the fundamental component in the frequency domain. It was reported before by Faez et al. that the ratio between the SH amplitude and the fundamental amplitude is independent from the excitation duration above three cycles [21]. We also found the enhancement of SH emission due to the S-D signal is still evident for a shorter transmitting pulse consisting of 6 cycles (figure. 3a).

The stimulation effect of the S-D signal on the SH response of the UCA can be even more effective if combined with the conventional contrast imaging techniques such as PI. Since the S-D signal is proportional to the second time derivative of the squared envelope of the transmit burst the phase of the S-D signals remains the same in both of the transmitted bursts in PI method. Moreover the phase of the SH emission from the UCA induced by the S-D signal will be the same. Therefore summing up the backscattered signal from the microbubbles in PI results in doubling the amplitude of the SH oscillation while removing the linear fundamental signals. Excitation bursts with rectangular envelopes were used by Needles et al for SH imaging together with PI resulting in $15 \mathrm{~dB}$ enhancement in the CTR compared with fundamental imaging [3]. Such an increase in the SH response of the microbubbles using waves with rectangular envelopes together with PI technique can be explained by the stimulation effect of the S-D signal on the SH emission. Figure 5 compares the spectrum of the backscattered signals from tissue mimicking phantom and the UCA for different modalities used in this study: B-mode, PI-mode and AM-mode. The PI technique reduces the linear fundamental echo from the tissue mimicking material to the noise level while enhances the nonlinear SH components scattered by UCA only. The AM method is not as powerful as the PI technique in tissue cancelation which might be due to the increased nonlinearity generated during propagation of the acoustic wave in the tissue at high frequencies [15] as well as the saturation of the higher pressure wave in AM method. In vitro results (figures. 5 and 6) demonstrates that maximum enhancement in CTR is reached using PI (up to $28 \mathrm{~dB}$ ).

\section{CONCLUSION}

The low frequency self-demodulation acoustic signal resulting from nonlinear propagation of the ultrasound wave, stimulates the subharmonic response of ultrasound contrast agents. The microbubbles are pushed towards the buckling regime by the self-demodulation signal resulting in enhanced subharmonic emission. Using the S-D signal a real time nonlinear contrast imaging method on an array based high frequency ultrasound system was demonstrated. Such a new nonlinear contrast detection method offers improved performance over a larger depth and a better CTR at high frequencies compared with the conventional linear and nonlinear contrast methods.

\section{ACKNOWLEDGMENT}

This research was supported by the Center for Translational Molecular Medicine and the Netherlands Heart Foundation (PARISK).

\section{REFERENCES}

[1] Staub D, Patel M B, Tibrewala A, Ludden D, Johnson M, Espinosa P, Coll B, Jaeger K A, Feinstein S B, "Vasa vasorum and plaque neovascularization on contrast-enhanced carotid ultrasound imaging correlates with cardiovascular disease and past cardiovascular events," Stroke 41,41-7 (2010). 
[2] Eisenbrey J R, Dave J K, Halldorsdottir V G, Merton D A, Machado P, Liu J B, Miller C, Gonzalez J M, Park S, Dianis S, Chalek C L, Thomenius K E, Brown D B, Navarro V and Forsberg F, "Simultaneous grayscale and subharmonic ultrasound imaging on a modified commercial scanner," Ultrasonics 51, 890-897 (2011).

[3] Needles A, Arditi M, Rognin N G, Mehi J, Coulthard T, Bilan-Tracey C, Gaud E, Frinking P, Hirson D and Foster F S, "Nonlinear contrast imaging with an array based micro-ultrasound system," Ultrasound Med. Biol. 36, 2097-2106 (2010).

[4] Burns P N, "Harmonic imaging with ultrasound contrast agents," Clinical radiology 51, 50-5 (1996).

[5] Simpson D H, Chin C T and Burns P N, "Pulse inversion Doppler: a new method for detecting nonlinear echoes from microbubble contrast agents," IEEE Trans Ultrason Ferroelectr Freq Control 46, 372-82 (1999).

[6] Vos H J, Goertz D E, van der Steen A F W and de Jong N, "Parametric array technique for microbubble excitation," IEEE transactions on ultrasonics, ferroelectrics, and frequency control 58, 924-34 (2011).

[7] Sarkar K, Shi W T, Chatterjee D and Forsberg F, "Characterization of ultrasound contrast microbubbles using in vitro experiments and viscous and viscoelastic interface models for encapsulation," J Acoust Soc Am 118, 539-50 (2005).

[8] Goertz D E, Frijlink M E, Tempel D, Bhagwandas V, Gisolf A, Krams R, de Jong N and van der Steen A F W, "Subharmonic Contrast Intravascular Ultrasound for Vasa Vasorum Imaging," Ultrasound Med. Biol. 33, 1859-72 (2007).

[9] Forsberg F, Shi W T and Goldberg B B, "Subharmonic imaging of contrast agents," Ultrasonics 38, 93-8 (2000).

[10] Leighton T G, [The Acoustic Bubble], Academic Press Ltd, London, (1994).

[11] Shankar P M, Krishna P D and Newhouse V L, "Subharmonic backscattering from ultrasound contrast agents," J. Acoust. Soc. Am. 106, 2104-10 (1999).

[12] Katiyar A and Sarkar K, "Excitation thresholds for subharmonic response of ultrasound contrast microbubbles," J. Acoust. Soc. Am. 130(5), 3137-3147 (2011.

[13] Faez T, Renaud G, Defontaine M, Calle S and De Jong N, "Dynamic manipulation of the subharmonic scattering of phospholipid-coated microbubbles," Physics in medicine and biology 56, 1-15 (2011).

[14] Biagi E, Breschi L, Vannacci E and Masotti L, "Subharmonic emissions from microbubbles: effect of the driving pulse shape," IEEE transactions on ultrasonics ferroelectrics and frequency control 53, 2174-82 (2006).

[15] Needles A, Graba J, Bilan C, Coulthard T, Hirson D, Foster FS, GoertzDE, Stapleton S, "A new real-time highfrequency subharmonic contrast mode for mouse imaging," Proc. IEEE 9854296, 1069-72 (2007).

[16] Foster FS, Mehi J, Lukacs M, Hirson D, White C, Chaggares C, Needles A, "A new 15-50 MHz array-based microultrasound scanner for preclinical imaging," Ultrasound Med Biol. 35, 1700-08 (2009).

[17] Averkiou M, Lee Y S and Hamilton M F, "Self-demodulation of amplitude- and frequency-modulated pulses in a thermoviscous fluid," J Acoust Soc Am 94, 2876-83 (1993).

[18] Vos H J, Goertz D E and de Jong N, "Self-demodulation of high-frequency ultrasound," J Acoust Soc Am 127, 1208-17 (2010).

[19] Hamilton M F and Morfey C L, [Model equations], Nonlinear Acoustics Academic, San Diego, (1998).

[20] Marmottant P, van der Meer S, Emmer M, Versluis M, de Jong N, Hilgenfeldt S and Lohse D, "A model for large amplitude oscillations of coated bubbles accounting for buckling and rupture," J. Acoust. Soc. Am. 118, 3499-505 (2005).

[21] Faez T, Emmer M, Docter M, Sijl J, Versluis M and De Jong A, "Characterizing the subharmonic response of phospholipid-coated microbubbles for carotid imaging," Ultrasound Med. Biol. 37, 958-70 (2011).

[22] Conversano F, Franchini R, Casciaro S, "Characterization of microbubble contrast agents for echographic imaging through time-scheduled size distribution measurements," Sensors and Transducers J. 9, 21-27 (2010).

[23] Teirlinck C J, Bezemer R A, Kollmann C, Lubbers J, Hoskins P R, Fish P, Fredfeldt K E, Schaarschmidt U G, "Development of an example flow test object and comparison of five of these test objects, constructed in various laboratories," Ultrasonics 36, 653-60 (1998). 